\title{
Numerical Analysis of Ratcheting Under Bending- Membrane Loading Conditions
}

\author{
Sajib Kumar Nath*, Md. Makfidunnabi and Md Abdullah Al Bari \\ Department of Mechanical Engineering, Khulna University of Engineering \& Technology, Khulna-9203, BANGLADESH
}

Received: March 25, 2020, Revised: April 02, 2020, Accepted: April 03, 2020, Available Online: April 04, 2020

\begin{abstract}
Ratcheting is a vital failure mode when dynamic loading is present in the scenario and it can lead to fatigue or incremental collapse if not restricted. The purpose of this study is to propose a ratchet diagram for primary bending and secondary membrane loading conditions. For this, a finite element analysis model of a rectangular beam is prepared and solved by the numerical analysis software 'ABAQUS'. The FEA model is validated by carried out a dynamic nonlinear elastic-plastic analysis with the analytical solution of Yamashita et al. for similar loading conditions. The ratchet occurrence conditions plotted in a non-dimensional stress parameter plot similar to the Bree diagram. The findings suggest that secondary stress rises for the occurrence of ratchet conditions as primary stress decreases. It also found a strong frequency dependency feature. The nature of the input frequency of cyclic loading in the proposed ratchet diagram has been discussed in terms of dynamic displacement over static displacement in the change of non-dimensional frequency of the loading.
\end{abstract}

Keywords: Ratcheting; FEA; Membrane Loading; Bending Loading; Frequency Effect.

This work is licensed under a Creative Commons Attribution-NonCommercial 4.0 International

\section{Introduction}

Ratcheting is defined as the phenomenon where the cyclic accumulation of inelastic deformation occurs if the stress applied is high enough to make the structure yield due to a cyclic stressing with non-zero mean stress [1]. It is a special kind of cyclic deformation behavior that occurs in materials as well as structures where stresses are greater than the yield stress of the material [2]. Many types of engineering structures are subjected to cyclic loading. So, ratcheting is a key point that is considered during designing those structures for predicting safety and fatigue life of them, because there are many components where the combined effect of several applied stresses can exceed their yield stress. For those components, accurate prediction of ratcheting behavior is necessary as the wrong prediction can lead to a catastrophic failure. Under certain conditions, a correct combination of steady primary stress and cyclic thermal stress leads to ratcheting. This mechanism of ratcheting under cyclic thermal stress was investigated by Miller [3], Burgreen [4] and Bree [5]. From the Bree diagram, different loading sequences are found including steady thermal cyclic primary, in-phase cyclic thermal and primary stress, out-of-phase cyclic thermal and primary stress [6]. It is also found from the Bree diagram that steady thermal and cyclic primary stress is not accountable for ratcheting.

Ohno et al. [7] stated that "ratcheting happening under uniaxial cyclic loading with the non-zero mean stress is noted to uniaxial ratcheting, which is most fundamental and has been studied in many works". Cyclic tension is a very common phenomenon found in many engineering components. Under asymmetrical loading, the component faces cyclic creep. If cyclic load with a constant stress amplitude and non-zero mean stress is applied to the body, the total strain developed within the body becomes so large that the original shape of the structure may change. So uniaxial ratcheting can be a subject of further investigation especially when there are not lots of researchers worked on this particular topic.
In the present study, a ratchet diagram has been proposed for various combinations of primary bending and secondary membrane loading conditions. At first, a FEM model of a rectangular beam has been made and validated with the theoretical model proposed by Yamashita et al. [8] for similar loading conditions. Finally, when the ratchet occurrence condition has been obtained for various frequencies, the effect of frequency has been characterized and discussed.

\section{Theory of Ratcheting}

Ratcheting is an important design criterion for the structures subjected to cyclic loadings. So, researches regarding the effects of ratcheting have been being done for a longer period of time. Researchers are trying to find out the effects caused by ratcheting on different materials and structures. Some of the relevant studies are important for the current study.

T. M. Mulcahy [9] investigated the effect name 'Bauschinger effect' with the help of thermal ratcheting upon a beam element. He investigated analytically for a beam element with a linear temperature variation across the solid crosssection of a rectangular beam. Finally, he tabulated specific results for materials and operating conditions associated with liquid metal breeder reactors.

T. H. Hyde, B. B. Sahari, and J. J. Webster [10] adopted the finite element method to investigate the behavior of thin tubes due to thermal ratcheting. The thin tubes were subjected to steady, internal pressure and cyclic, linear, through-thickness temperature distributions. They used an elastic-perfectly plastic material model for the analysis and related uniaxial behavior with multi-axial via von Mises yield criterion and PrandltReuss flow rule. They found for an axially constrained tube that "if thermal loading is high enough then yielding occurs through the whole of the wall thickness simultaneously in each half cycle".

G.Z. Kang, Y.G. Li, J. Zhang, Y.F. Sun and Q. Gao [11] experimented on 25CDV4.11 steel and SS304 stainless steel for 
ratcheting and failure behaviors under uniaxial cyclic tests. The experiment was conducted at room temperature. In the experiment, they first observed the cyclic hardening/softening features of the materials under uniaxial strain cycling. Finally, they observed for the ratcheting and failure behavior of those materials.

Md Abdullah Al Bari, Ryota Sakemi, Yamato Katsura and Naoto Kasahara [12] experimented with a rectangular beam under dynamic loading like seismic loading. They investigated for determining the conditions for the occurrence of ratcheting and collapse failure. The analogy between thermal ratcheting and dynamic ratcheting was considered in the analysis. A nonlinear dynamic finite element method was used to analyze the beam. Finally, they obtained experimental observations for ratcheting which they used to validate the analytical predictions and proposed a failure mode map.

K.S. Basaruddin and L.C. Wooi [13] experimentally investigated the uniaxial ratcheting characteristics of mild steel at room temperature. They conducted cyclic tension tests along with conventional monotonic, cyclic and ratcheting tests. Finally, they observed that "at the same stress amplitude (mean stress), the ratcheting strain increase as mean stress (stress amplitude) increased.

The simultaneous presence of constant primary stress and cyclic secondary stress in a uniaxial stress field can occur four types of ratcheting. Yamashita et al. [8] proposed a simplified evaluation method of ratcheting under primary bending and secondary membrane stress to develop an evaluation method for bending-membrane types of ratcheting. They calculated the dissipated energy of a beam due to bending-membrane loading using modified yield stress for the beam. They obtained that ratcheting will occur of a rectangular beam if

$$
\frac{2 X}{3}+\frac{Y}{2}>1
$$

where $\mathrm{X}$ and $\mathrm{Y}$ are non-dimensional stress parameters [8]. Yamashita et al. proposed the following ratchet diagram (Fig. 1) by the analytical solution of a rectangular beam. Fig. 2 showed the loading and analysis condition of Yamashita et al.'s model.

There are other ratchet diagrams proposed for different loadings too. Ratchet diagram by Bree for membrane-bending loading conditions, Yamashita et al. [8] bending-bending theoretical ratchet diagram and subsequent numerical analysis of Bari et al. [12] are among them. The current study is based on the finite element analysis of Yamashita et al.'s theoretical bending-membrane ratchet model.

\section{Methodology}

In the current study, finite element analysis was used by numerical analysis software 'Abaqus'. The analysis conditions are shown in Table 1. The loading and constraint of the model are shown in Fig. 3. One end of the beam model was made fixed and at the other end movement along the vertical direction was restricted. The element type was chosen plane stress as the stress along thickness direction was insignificant. The elasticperfectly plastic material model was used which is similar to Yamashita et al.'s theoretical model. In the ratcheting analysis, a rectangular beam of $140 \mathrm{~mm}$ in length, $13 \mathrm{~mm}$ in width and 6 $\mathrm{mm}$ in height was used.

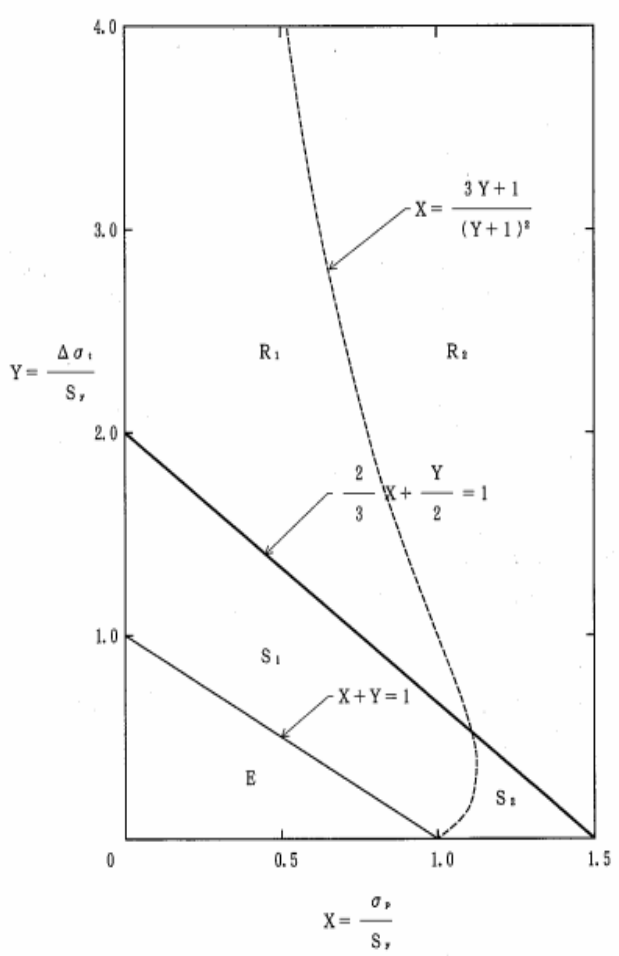

Fig. 1 Theoretical bending-membrane Ratchet diagram by Yamashita et al. [8]

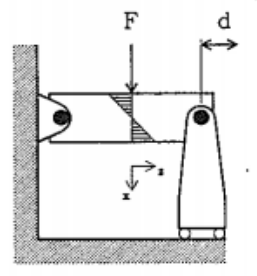

(a) Structural Model

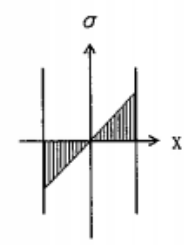

(b) Primary Stress

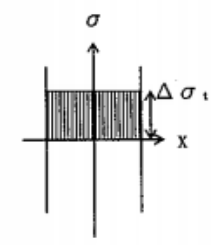

(c) Secondary Stress
Fig. 2 Loading and analysis condition of Yamashita et al.'s theoretical model [8]

Table 1 Finite element analysis conditions

\begin{tabular}{ll}
\hline FEA platform & Abaqus ver.6.14 \\
\hline Analysis type & $\begin{array}{l}\text { Dynamic elastic-plastic analysis with } \\
\text { large deflection on }\end{array}$ \\
Element type & Plane stress (CPS4R) \\
Number of elements & 2340 \\
& \\
Input excitation & Displacement at the free end \\
Material properties & Lead (Pb) \\
& $\begin{array}{l}\text { Density } \\
\text { Young's }\end{array}$ \\
& modulus (E) $\quad 16 \mathrm{GPa}$ \\
& $\begin{array}{l}\text { Poisson's ratio } \quad 0.42 \\
\text { Yield's stress } \quad 5 \mathrm{MPa}\end{array}$ \\
& Elastic perfectly plastic \\
\hline
\end{tabular}




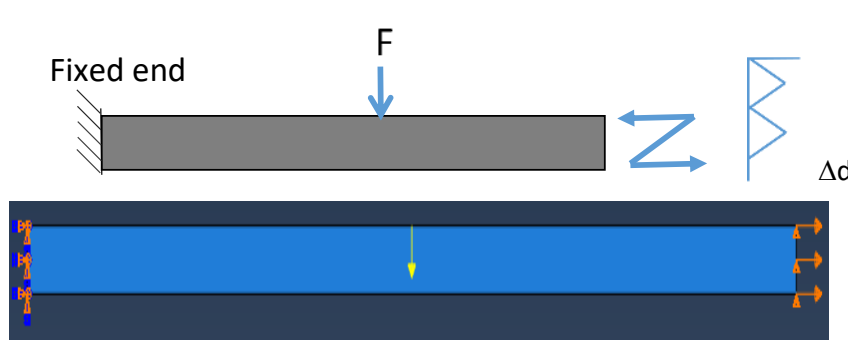

Fig. 3 Loading and constraint of the model

Ratcheting is observed under two loading conditions; primary and secondary loading. Primary loading is a load controlled loading and secondary loading is a displacement controlled loading. In this experiment, a concentrated load was used as primary loading and a cyclic displacement in axial direction was used as secondary loading similar to Yamashita's model. Concentrated load acts on the upper face of the beam. It acts downward along the midpoint of the beam. Displacement acts on the free end of the beam. It acts along the beam axis. A triangular shape of displacement was used with 10 cycles.

\section{Results and Discussions}

Ratcheting can be understood from strain vs time diagram shown in Fig. 4. When ratcheting occurs, a gradual increase in strain is noticed in the diagram with time. If no gradual increase in strain with time is found, then it is evident that no ratcheting has occurred.

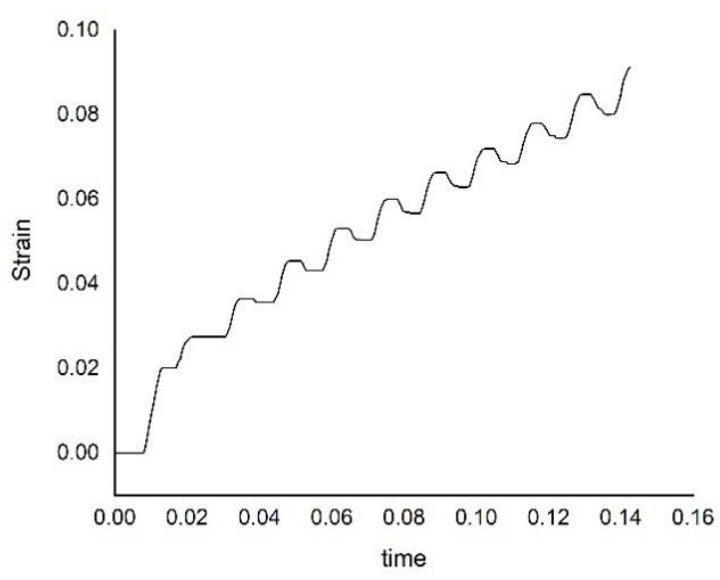

Fig. 4 Strain vs time diagram

As no experiment has been done in current research, so the numerical result is compared with Yamashita et al.'s theoretical ratchet diagram for validation purposes and shown in Fig. 5. From the comparison, it had seen that the numerical result followed an almost similar trend to the theoretical results. But a little deviation was also found at several points. The maximum deviation found was about $5 \%$.

The ratcheting occurrence condition has been determined for different frequencies and plotted in terms of nondimensional stress parameter X, Y diagram. Here, X is the nondimensional primary stress parameter that can be expressed as

$X=\frac{\sigma^{d}}{s_{y}}$

and $\mathrm{Y}$ is the non-dimensional secondary stress parameter that can be expressed as
$Y=\frac{\sigma^{m}}{s_{y}}$

Here $\sigma^{d}$ is the bending stress due to concentrated load and $\sigma^{m}$ is the bending stress due to secondary membrane load. $S_{y}$ is the yield stress. In the diagram, $f_{n}$ stands for the natural frequency of the beam. For example, $1.5 f_{n}$ means the applied frequency is 1.5 times the natural frequency of the beam. The stresses $\sigma^{d}$ and $\sigma^{m}$ is calculated by using the beam formula. Like the bending stress is calculated by using the following formula

$\sigma^{d}=\frac{3 F l}{b h^{2}}$

where $F$ is the applied concentrated load, $l$ is the length of the beam and $b, h$ stands for beam width and height correspondingly.

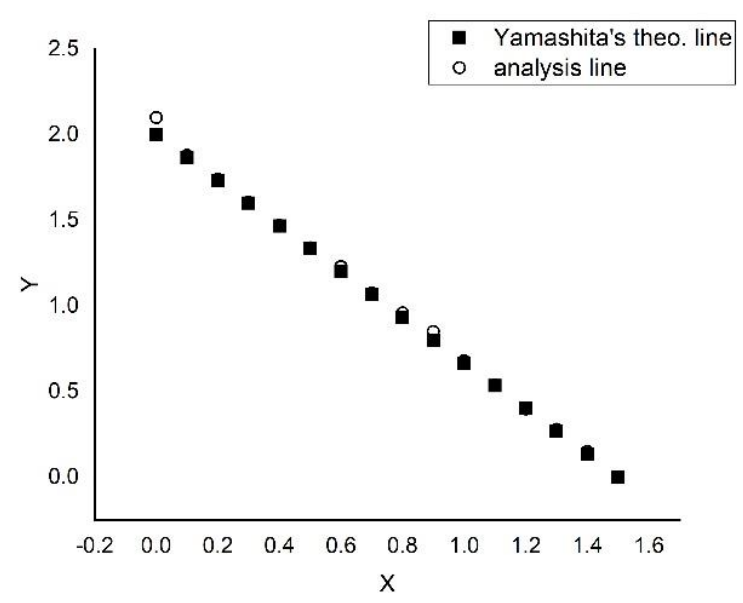

Fig. 5 Validation of numerical model with the theoretical model

The non-dimensional primary stress parameter $\mathrm{X}$ in the proposed ratchet diagram was plotted in abscissa, and the nondimensional secondary stress parameter $\mathrm{Y}$ was plotted in ordinate. The $\mathrm{X}$ values varied from 0.4 to 1.5 and $\mathrm{Y}$ was varied to find the state of ratchet occurrence condition for every $\mathrm{X}$ values. By changing the concentrated force $F$, the $X$ values were varied, and Table 2 displayed the corresponding $\mathrm{X}$ values for concentrated force $F$.

Table 2 Forces corresponding to the values of $\mathrm{X}$

\begin{tabular}{cc}
\hline $\mathrm{F}(\mathrm{N})$ & $\mathrm{X}$ \\
\hline 2.229 & 0.4 \\
3.343 & 0.6 \\
4.457 & 0.8 \\
5.5714 & 1.0 \\
6.686 & 1.2 \\
7.8 & 1.4 \\
\hline
\end{tabular}

After getting the $\mathrm{X}$ and corresponding $\mathrm{Y}$ values, a ratchet diagram is plotted in Fig. 6. This ratchet diagram was plotted for natural frequency, which means the frequency of input secondary loading was the same as the natural frequency of the beam. From this graph, it has shown that Y decreases as $\mathrm{X}$ increases, but the graph's slope has been varied in various regions. 


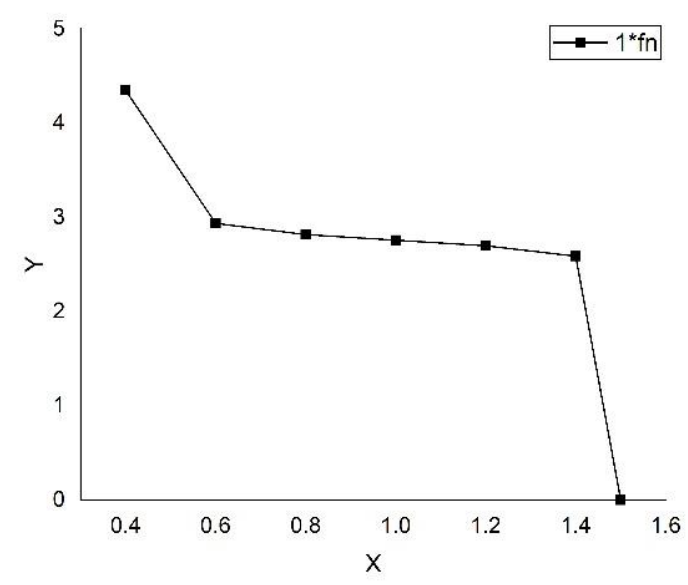

Fig. 6 Non-dimensional ratchet diagram for natural frequency

After completing the ratchet analysis and plotting all data in a non-dimensional stress plot similar to Yamashita's model, the final proposed ratchet diagram is obtained.

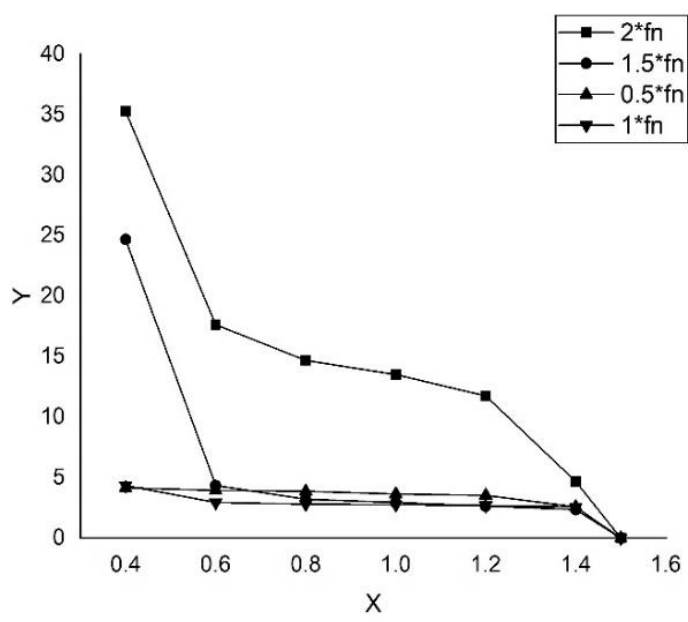

Fig. 7 Proposed ratchet diagram for bending-membrane loading conditions

In the proposed ratchet diagram, different lines representing the ratchet occurrence condition at different applied frequencies. Fig. 7 shows similar patterns of ratchet occurrence conditions as Yamashita et al.'s ratchet diagram. From this figure, it has shown that the secondary stress varies considerably for different levels of frequency for the occurrence of ratchet for particular primary stress. It can be shown that the lowest line in the proposed ratchet diagram was for $1 f_{n}$, for the same ratchet incidence conditions the outermost line was for $2 f_{n}$ and other lines were for $0.5 \& 1.5 f_{n}$ lies between those two lines. So it can be said that, in the case of ratchet occurrence, there was a strong frequency effect.

The effect of frequency on ratcheting occurrence condition can be explained with the help of the amplification factor graph as shown in Fig. 8. The plot's horizontal axis represents the nondimensional frequency that is obtained by dividing the frequency of input by the normal beam frequency. The vertical axis represents the dynamic displacement over static displacement, which in other words, the amplification of displacement due to dynamic loading. From the amplification factor graph, there are three distinct regions can be observed. It is evident that dynamic displacement and static displacement were almost the same for non-dimensional frequency range 0.25-0.5. From the plotting, the dynamic displacement increases when the frequency range increases from 0.5 and becomes maximum at the value of 1.0 which is called the resonance region. At the resonance region, a sudden increase in displacement was found. This sudden increase of displacement indicated that at natural frequency dynamic loading amplified and due to this, a small secondary loading could enough to cause ratcheting and that was why the lowermost line in the ratchet diagram was for natural frequency. On the other hand for higher non-dimensional frequency range, dynamic displacement decreases gradually and after 1.5 nondimensional frequency, lower values of dynamic displacement were found and due to this, the effect of dynamic loading decreases at this frequency ranges. Because of this effect, the outermost lines in the ratchet diagram were for higher frequencies.

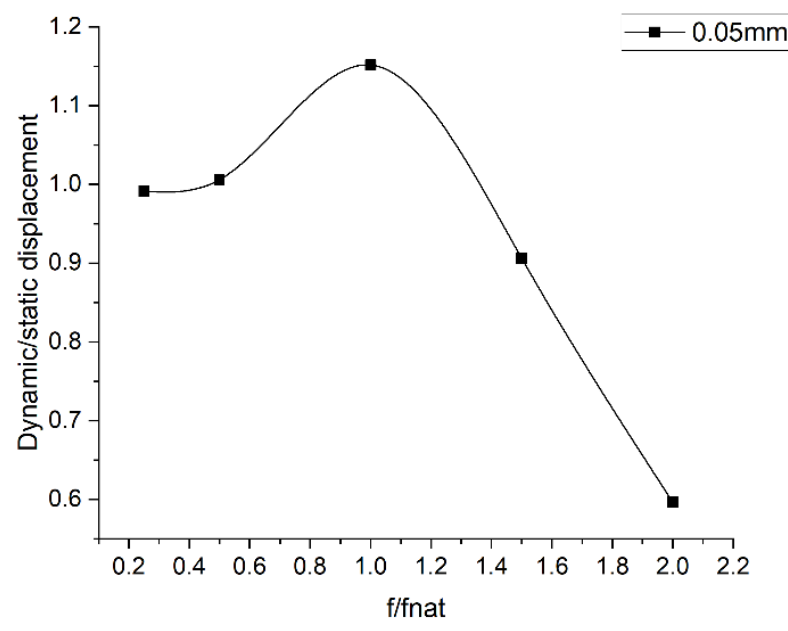

Fig. 8 Amplification factor graph

\section{Conclusion}

In this study, a dynamic non-linear elastic-plastic analysis was carried out on a rectangular beam for bending-membrane loading conditions. An FEA model was prepared and validated by Yamashita et al.'s analytical result. The analytical and numerical results showed a reasonable degree of agreement. The numerical model has then been used to propose a ratchet diagram for bending-membrane loading conditions and to investigate the effects of the frequency of input cyclic loading on ratchet occurrence. Finally, a ratchet diagram has been proposed for bending-membrane loading conditions. The Frequency dependency characteristics have also been observed in the ratchet diagram. The frequency effect was analyzed by the amplification factor graph and it has been found that the natural frequency was more dangerous and higher frequencies were safer for the structure in terms of ratcheting.

\section{References}

[1] Chen X, Chen X, Yu D, Gao B. Recent progresses in experimental investigation and finite element analysis of ratcheting in pressurized piping. International Journal of Pressure Vessels and Piping. 2013 Jan 1;101:113-42. 
[2] Shariati M, Hatami H, Yarahmadi H, Eipakchi HR. An experimental study on the ratcheting and fatigue behavior of polyacetal under uniaxial cyclic loading. Materials \& Design. 2012 Feb 1;34:302-12.

[3] Miller DR. Thermal-stress ratchet mechanism in pressure vessels. Journal of Basic Engineering. 1959 Jun 1;81(2):190-4.

[4] Burgreen D. Structural growth induced by thermal cycling. Journal of Fluid Engineering. 1968 December; 90(4): 469-75.

[5] Bree J. Elastic-plastic behaviour of thin tubes subjected to internal pressure and intermittent high-heat fluxes with application to fast-nuclear-reactor fuel elements. Journal of strain analysis. $1967 \mathrm{Jul}$ 1;2(3):226-38.

[6] Ng HW, Moreton DN. Bree diagrams for alternative loading sequences(for identifying boundaries of strain behavior in thermal and pressure loading). Engineering approaches to high temperature design(A 84-37877 1739). Swansea, Wales, Pineridge Press, 1983,. 1983:279312.

[7] Mizuno M, Mima Y, Abdel-Karim M, Ohno N. Uniaxial ratchetting of 316FR steel at room temperature-Part I: experiments. J. Eng. Mater. Technol.. 2000 Jan $1 ; 122(1): 29-34$..
[8] Yamashita T, Tsukimori K, Nagata T. Development of the simplified evaluation method for ratchetting due to primary bending stress and secondary membrane stress. Commercial Property. PNC ZN9410. 1992 May: 92-139.

[9] Mulcahy TM. Thermal ratcheting of a beam element having an idealized bauschinger effect. Journal of Engineering Materials and Technology, 98(3). 1976 July: 264-71.

[10] Hyde TH, Sahari BB, Webster JJ. The effect of axial loading and axial restraint on the thermal ratchetting of thin tubes. International journal of mechanical sciences. 1985 Jan 1;27(10):679-92.

[11] Kang GZ, Li YG, Zhang J, Sun YF, Gao Q. Uniaxial ratcheting and failure behaviors of two steels. Theoretical and Applied Fracture Mechanics. 2005 May 1;43(2):199209.

[12] Al Bari MA, Sakemi R, Katsura Y, Kasahara N. Proposal of Failure Mode Map Under Dynamic LoadingRatcheting and Collapse. Journal of Pressure Vessel Technology. 2018 Oct 1;140(5).

[13] Basaruddin KS, Wooi LC. Uniaxial ratcheting of mild steel under cyclic tension. In International Conference on Applications and Design in Mechanical Engineering (ICADME), Batu Ferringhi, Penang, Malaysia 2009 Oct (pp. 6E-1 -4). 